D.O.I.: $10.3895 / \mathrm{S} 1808-04482007000400002$

\title{
TIPOLOGIA DE REDES, ESTRATÉGIA E INSERÇÃO COMPETITIVA: UM ESTUDO EM UM CONSÓRCIO DE EMPRESAS DE BASE TECNOLÓGICA
}

\section{TIPOLOGY OF NETS, STRATEGY AND INSERTION COMPETITIVE: AN STUDY OF CASE IN CONSORTIUM ENTERPRISES OF TECHNOLOGICAL BASE}

\author{
Patrícia Trindade Caldas ${ }^{1}$; Ana Cecília Feitosa de Vasconcelos ${ }^{2}$; Gesinaldo Ataíde Cândido ${ }^{3}$ \\ ${ }^{1}$ Universidade Federal da Paraíba - UFPB - João Pessoa - Brasil ptcaldas@yahoo.com.br \\ ${ }^{2}$ Universidade Federal de Campina Grande - UFCG - Campina Grande - Brasil \\ acvasconcelos@gmail.com \\ ${ }^{3}$ Universidade Federal de Campina Grande - UFCG - João Pessoa - Brasil gacandido@uol.com.br
}

\begin{abstract}
Resumo
As transformações nos contextos ambientais, políticas, sociais, econômicas e mercadológicas ocorridas no mundo dos negócios desafiam as organizações na busca por melhores práticas que as sustentem frente a competitividade acirrada do mercado globalizado atual. As redes de empresas surgem justamente com o intuito de viabilizarem tal objetivo, onde embasadas em principios básicos de cooperação, interação, ajuda mútua e confiança agem de forma estratégica em prol de uma sustentação competitiva coletiva. Este artigo tem como objetivo analisar um consórcio de empresas de base tecnológica, a partir das características de redes interorganizacionais propostas por Hoffmann et al. (2004), comparando a situação atual da rede com a situação ideal para o setor que participam empresas de base tecnológica com atuação em Tecnologia da Informação e Comunicação (TICs). Além disso, verifica o alinhamento entre as características da rede com o tipo de estratégia adotada, utilizando tipologia de estratégias, propostas por Miles e Snow (1978). Este trabalho se caracteriza como exploratório e descritivo conduzido sob a forma de estudo de caso, empregando como técnicas a pesquisa bibliográfica e a coleta de dados secundários e primários referentes à dados atualizados sobre a rede PBTech. Os resultado mostraram como empresas de pequeno e médio porte, ao se unirem em rede, e se configurando adequadamente em termos de estrutura e estratégia frente à um propósito maior coletivo podem se diferenciar competitivamente no mercado.
\end{abstract}

Palavras-chave: redes de empresas, estratégia, competitividade.

\section{Introdução}

O atual ambiente de negócios é marcado por incertezas e turbulências constantes, que obriga as organizações a buscarem, cada vez mais, formas de se adaptarem e encontrarem as melhores 
práticas que viabilize as condições ideais de atuação e assim, garantam a sua sobrevivência e o seu desenvolvimento.

Nessa nova perspectiva surgem os estudos sobre as redes organizacionais, assim como pela sua aplicação utilizada como recurso estratégico, na medida em que aumenta a eficiência operacional e o desenvolvimento de novas competências, viabilizado pelos processos de colaboração e cooperação estabelecidos pelas empresas participantes de uma determinada rede. Para Balestrin e Vargas (2005), a configuração em rede consiste em uma forma eficaz para as empresas alcançarem competitividade nos mercados por meio de complexo ordenamento de relacionamentos, em que as firmas estabelecem inter-relações.

Nesse sentido, o objetivo do artigo é analisar um consórcio de empresas de base tecnológica, a partir das características de redes interorganizacionais propostas por Hoffmann et al. (2004), comparando a situação atual da rede com a situação ideal para o setor que incorpora empresas de base tecnológica com atuação em Tecnologia da Informação e Comunicação (TICs). Além disso, verifica o alinhamento entre as características da rede com o tipo de estratégia adotada, utilizando tipologia de estratégias, propostas por (MILES; SNOW, 1978).

Para a consecução deste, foram utilizadas como técnicas a pesquisa bibliográfica e a coleta de dados secundários e primários referentes à dados atualizados sobre a rede PBTech. Este estudo caracteriza-se como descritivo conduzido sob a forma de estudo de caso.

Como forma de favorecer o entendimento, o artigo explicita, além desta parte introdutória, o referencial teórico que está assim apresentado: Redes, que se subdivide em Tipologia de Redes e a Tipologia de Hoffmann et. al.(2004) ; Estratégia, que apresenta um subtópico: Tipologia Estratégica de Miles e Snow (1978). Em seguida estão apontados os procedimentos metodológicos, a caracterização da rede, a análise dos resultados, as considerações finais e, por fim as referências bibliográficas que deram suporte a consecução deste trabalho.

\section{Redes}

O termo rede não é novo e apresenta diversos significados e aplicações nos mais variados contextos. De acordo com Nohria (1992) foi nos anos 30 que houve o despertar para sua utilização sistemática da idéia, dos conceitos e aplicações de rede pelas mais variadas áreas do conhecimento, partindo do princípio de que o homem é um ser eminentemente social e necessita durante toda a sua existência manter diversos tipos de relacionamentos e interações com outras pessoas. Este tipo de comportamento social pode ser estendido para os grupos sociais, dentro do princípio de que estes grupos são formados por pessoas. Os princípios fundamentais da aplicação dos conceitos de Redes 
são: a interação, o relacionamento, a ajuda mútua, o compartilhamento, a integração e a complementaridade (CÂNDIDO, 2001).

Com o passar do tempo, esse referido termo adquiriu sentido mais abstrato, recebendo diversas definições, advindas de diversos autores e estudiosos e, sob diversas perspectivas de análise. No campo de estudos das ciências sociais, o termo rede designa um conjunto de pessoas ou organizações interligadas direta ou indiretamente (MARCON; MOINET, 2000). Para Castells (1999) a intensidade e a freqüência da interação dos atores sociais são maiores se esses atores forem 'nós' de uma rede do que se não pertencerem a ela, e de acordo com Fayard (2000) as redes intensificam a interação, promovendo redução espaço-temporal nas inter-relações dos seus atores, como fatores altamente estratégicos para a competitividade das organizações do século XXI.

$\mathrm{Na}$ formação de redes entre empresas, existe a possibilidade destas configurarem-se como redes flexíveis de pequenas e médias empresas, como clusters de empresas (agrupamentos), ou como redes de cooperação, geralmente como organizações virtuais, ou ainda como as chamadas "supply chain management" ou gerenciamento da cadeia de suprimentos (OLAVE; AMATO NETO, 2001).

Diante de sua relevância e abrangência, as redes podem ser estudadas a partir de diversas perspectivas de análise, oferecendo uma preciosa base de conceituação. Nesse sentido, diversos autores sugeriram algumas tipologias no intuito de simplificar e facilitar a sua compreensão e estudo.

\subsection{Tipologia de redes}

Diante da variedade de conceituação e definição acerca de redes, em várias áreas do conhecimento, surgem várias formas de classificar as redes, desde as mais genéricas até as mais específicas. A partir de alguns atributos básicos, alguns autores criaram tipologias no intuito de classificar as redes e assim, contribuir para uma melhor compreensão acerca das ações (atitudes e comportamentos) dos atores organizacionais. Para alguns autores, as redes podem se configurar entre extremos, não significando que existam tipos ideais de rede, mas que diferentes tipos e situações podem levar a diferentes acordos e interações entre as empresas.

Frente à diversidade de tipologias e classificações para as redes de empresas, optou-se pela tipologia de Hoffman et. al. (2004), para a consecução deste, uma vez que esta consegue abordar de forma prática e didática o que as outras tipologias abordam.

\subsubsection{Tipologia de Hoffmann et. al. (2004)}


A partir de uma revisão literária acerca dos principais textos relevantes sobre o tema, e da compilação e combinação de dois trabalhos empíricos, os autores Valmir Hoffmann, Franscesc Molina-Morales e Maria T. Martinez-Fernadez, propõem uma interessante tipologia de classificação das redes de empresas, explorando quatro características conforme a tabela 1 :

Tabela 1 - Característica e tipologia das redes

\begin{tabular}{cc}
\hline Indicadores & Tipologia \\
\hline Direcionalidade & Vertical \\
& Horizontal \\
\hline Localização & Dispersa \\
& Aglomerada \\
\hline Formalização & Base contratual formal \\
& Base não contratual \\
\hline Poder & Orbital \\
& Não orbital \\
\hline
\end{tabular}

Fonte: Hoffmann et. al. (2004)

O primeiro indicador está atrelado a direção das relações entre as partes, onde a rede, pode ser classificada como Vertical ou Horizontal. As redes verticais caracterizam-se pela colaboração entre empresas com produtos complementares ou em fases diferentes de uma mesma cadeia produtiva. Uma rede é considerada vertical quando realizada por empresas distintas, adotando a estratégia de especialização com o objetivo de alcançar eficiência coletiva nos processos a partir da rapidez de resposta das partes. Há presença da prática da terceirização de processos, quando estes não se configuram como agregadores de valor para o produto.

Já as Redes Horizontais, de acordo com Hoffmann et. al. (2004) são compostas por empresas que competem em termos de produtos e/ou mercados e que se juntam com o objetivo de obter ganhos pela união entre as partes por meio da cooperação em certos domínios, benefícios estes como a aprendizagem interorganizacional, a criação de novos mercados, suporte de custos e riscos em pesquisas e desenvolvimento de novos produtos, gestão da informação e de tecnologias, definição de marcas de qualidade, defesa de interesses, ações de marketing, implementações de exportação e de projetos de produtos e/ou processos. Assim, essas redes "favorecem a concentração de esforços, sem privar a liberdade de ação estratégica de seus membros" (BALESTRIN; VARGAS, 2004, p.209)

O segundo indicador está relacionado a dispersão geográfica das empresas na rede, podendo então ser classificada como redes Dispersas ou Aglomeradas. As redes Dispersas são aquelas que 
apresentam suas empresas participantes com certo afastamento geográfico, que interagem através de um processo logístico avançado que permite superar distâncias (HOFFMANN et al., 2004). Geralmente se unem com o objetivo de desenvolvimento e produção de produtos e serviços, ou para ampliar a presença de mercado. Já as redes aglomeradas territorialmente mantêm relações que muitas vezes se estendem além daquelas puramente comerciais, estimulando uma maior cooperação e confiança entre as organizações. Nesse tipo de rede é possível encontrar instituições de apoio empresarial, como universidades, centros de tecnologia, e instituições governamentais suportando as necessidades da rede.

A terceira dimensão diz respeito à formalização das redes, podendo ter suas estruturas classificadas como formais, através de uma base contratual, ou então, ser informais, de base não contratual. As de base contratual formal apresentam uma maior garantia em relação à comportamentos oportunistas das contra-partes, evitando lesões e custos. Geralmente os contratos estabelecem regras de conduta, direitos e deveres para as empresas interessadas, com a finalidade de obter um maior controle sobre suas ações. Já as de base não-contratual são formadas por empresas que "agem em conformidade com os interesses mútuos de cooperação, baseados, sobretudo, na confiança entre os atores" (BALESTRIN; VARGAS, 2004, p.209), havendo uma mistura das relações econômicas com as sociais. Como se percebe, a confiança aqui é um tributo inquestionável de ser apresentado, embora ocorra, geralmente dispositivos como as sanções coletivas, que envolvem membros que punem outras empresas que violam normas de grupo, ou objetivos.

E por último, a dimensão poder de decisão, se retratando a ocorrência de hierarquia de poder. A rede é orbital quando possui um centro de poder ao redor do qual as demais empresas circulam. Este detentor possui maior poder de decisão em relação aos destinos que a sua rede vai tomar que as demais empresas-membro. Na rede não-orbital, cada parte tem a mesma capacidade de tomada de decisão, onde agem e trabalham de maneira cooperada, sem a existência de um centro de poder e o processo de tomada de decisão acontece de forma participativa.

A classificação da tipologia de uma rede é importante para compreender as dinâmicas e interações existentes influenciadoras nos demais aspectos organizacionais relacionados com a estratégia a ser perseguida. Aliás, a própria formação em rede apresenta uma perspectiva estratégica, ao se considerar a configuração em rede como meio para alcançar um fim (BALESTRIN; VARGAS, 2004). A relação efetiva entre estrutura e estratégia é importantíssima para um maior monitoramento do ambiente e ajustamento de ofertas às mudanças em curso, e assim um gerenciamento mais eficaz em prol da vantagem competitiva dessas redes. Tal relacionamento é apoiado neste estudo. 


\section{Estratégia}

O conceito de estratégia foi evoluindo ao longo dos tempos, passando a incluir habilidades gerenciais visando algum controle, da empresa, sobre o ambiente envolto, tanto interno como externo, tanto de forma reativa como pró-ativa (CHILD, 1997). Assim, a escolha estratégica é considerada o principal elo entre a organização e o ambiente. (ANDREWS, 1965; CHILD, 1972; MILES E SNOW, 1978). Sua delimitação envolve percorrer uma cadeia de meios e fins, a partir da maximização de informações, da alocação de recursos e do arranjo político, visando o desvio de ameaças ou o aproveitamento de oportunidades ambientais.

Por se tratar de um campo de estudo bastante amplo, há uma variedade de definições e conceitos de estratégia na literatura, algumas visões semelhantes e complementares e outras visões divergentes. A despeito disso, Besanko et al. (2000) comentam que muitos possuem frases comuns como "metas de longo prazo", "políticas" que sugerem que estratégia está relacionada com decisões que a empresa toma e as conseqüências do seu sucesso ou falha.

Por focar em micro, pequenas e médias empresas (PME’s), Miles e Snow (1978) aborda uma interessante tipologia onde as estratégias e operações destas empresas são bastante dependentes da ação dos seus proprietários-gerentes. Pelas empresas da rede de cooperação, foco deste trabalho, se caracterizarem como tal porte, optou-se pela aplicação desta tipologia.

\subsection{Tipologia estratégica de Miles e Snow (1978)}

A tipologia de estratégias proposta por Miles e Snow (1978) tem atraído a atenção de pesquisadores em administração estratégica por se tratar de uma classificação mais abrangente e pela possibilidade de aplicação em pequenas empresas, possibilitando uma análise do comportamento organizacional, que independe do tamanho da organização.

Os autores abordam que uma organização eficaz estabelece processo contínuo de reavaliação de seus propósitos e alinhamento com o meio ambiente, adequando sua estrutura de tarefas, relacionamentos, processos de tomada de decisão e de controle.

Segundo Zahra e Pearce (1979), a força desta taxonomia é que ela especifica relacionamentos entre estratégia, estrutura e processos de uma forma que permite a identificação das organizações como todos integrados em interação com seus ambientes. Para tanto, as empresas componentes buscariam a sinergia através de estratégias específicas para funções principais, conforme explicitada na tabela 2, abaixo: 
Tabela 2 - Tipologia Estratégica de Miles e Snow

\begin{tabular}{|c|c|}
\hline $\begin{array}{c}\text { Tipologia } \\
\text { Estratégica }\end{array}$ & Caracterização \\
\hline $\begin{array}{l}\text { Estratégia } \\
\text { Defensiva }\end{array}$ & $\begin{array}{l}\text { As empresas que adotam este tipo de estratégia apresentam domínio produto-mercado } \\
\text { estreito, geralmente direcionadas para a produção, aplicando os conceitos da economia de } \\
\text { escala, melhorias constantes no processo de produção, com foco na qualidade, produtividade } \\
\text { e custos. }\end{array}$ \\
\hline $\begin{array}{l}\text { Estratégia } \\
\text { Prospectora }\end{array}$ & $\begin{array}{l}\text { Uma empresa que adota esta estratégia são criadoras de mudança e incerteza, lidando } \\
\text { diretamente com funções relacionadas a Pesquisa e Desenvolvimento (P\&D), e à inovação. } \\
\text { Procura novas oportunidades de negócios, buscando ampliar continuamente sua linha de } \\
\text { produtos/serviços através da inovação tecnológica (de produtos e processos), assim como a } \\
\text { aplicação das novas tecnologias de gestão, desenhando e projetando os novos processos de } \\
\text { trabalho e formas de gestão. }\end{array}$ \\
\hline $\begin{array}{l}\text { Estratégia } \\
\text { Analítica }\end{array}$ & $\begin{array}{l}\text { Uma empresa que segue esta estratégia geralmente opera em duas frentes: uma estável - de } \\
\text { forma rotineira e, outra em mudança - buscando constantemente novas idéias, como no caso } \\
\text { das empresas envolvidas com fornecimento de matéria-prima e componentes para a } \\
\text { produção e a distribuição dos produtos, este sendo considerado um importante canal para } \\
\text { captação de informações do ambiente externo da rede. }\end{array}$ \\
\hline $\begin{array}{l}\text { Estratégia } \\
\text { Reativa }\end{array}$ & $\begin{array}{l}\text { As empresas que adotam uma estratégia reativa percebem mudanças em seu ambiente mas } \\
\text { são incapazes de responder adequadamente, por não possuírem uma relação consistente } \\
\text { estratégia-estrutura, assim elas não possuem habilidades para responder efetivamente as } \\
\text { implicações das mudanças ocorridas no seu ambiente. }\end{array}$ \\
\hline
\end{tabular}

Fonte: Adaptado de Silva Filho e Cândido (2002)

A partir das considerações embasadas do referencial teórico partir-se-á para a busca de nivelamento entre estrutura de rede de cooperação no setor econômico de TICs e as estratégicas competitivas mais adequadas, baseado no estudo de caso referente a rede de cooperação de empresas PBTECH.

\section{Metodologia}

A pesquisa realizada pode ser caracterizada como um estudo exploratório e descritivo, conduzido sob a forma de estudo de caso, o qual procurou observar as características de um grupo de empresas e estabelecer relações entre as variáveis. Neste caso, identificou-se as tipologias de redes das empresas participantes da rede PBTech, a partir do modelo de Hoffmann et. al. (2004), associada à identificação de estratégias preponderantes adotadas pelas mesmas, baseadas na tipologia estratégica de Miles e Snow (1978). Para tanto, utilizou-se como técnica a pesquisa bibliográfica.

O processo de coleta de dados ocorreu através da análise documental, incluindo dados secundários referentes a diagnóstico do setor de TICs na Paraíba e estudo do setor na perspectiva 
de Arranjos Produtivos Locais. Como complemento foram obtidos dados primários para o estudo, através da realização de entrevistas com os gestores do consórcio, a partir de um roteiro prédefinido.

O tratamento dos dados foi realizado a partir de inferências realizadas sobre as respostas obtidas na aplicação do instrumento de pesquisa e as suas interrelações com a análise documental e com a base teórica e conceitual sobre os temas centrais do estudo, quais sejam: redes de empresas e estratégia empresarial.

\section{Caracterização da rede}

A Paraíba abriga hoje muitas empresas de base tecnológica (EBTs) e se destaca nacionalmente e internacionalmente pelo seu potencial inovador decorrente do pólo tecnológico instalado em sua região, que conta com uma excelente infra-estrutura e ainda tem o apoio de diversas instituições de pesquisa e desenvolvimento.

Dentre as várias iniciativas decorrentes da oportunidade gerada pelo ambiente econômico favorável deste setor na Paraíba, a PBTech se configura como um consórcio entre empresas high tech que tem como objetivo principal promover a internacionalização das empresas do pólo de Tecnologia da Informação - TI da Paraíba.

Criado em dezembro de 2002, o Consórcio de Exportação de Software PBTech reúne 11 empresas de base tecnológica localizadas em Campina Grande e de João Pessoa, que juntas pretendem intensificar o processo de divulgação, comercialização local e nacional, além da exportação dos produtos fabricados no Estado (PBTECH, 2007). Desde a sua criação foram gerados um total de 54 empregos e mantidos 338 profissionais em seus postos de trabalho, com estimativas de geração de 130 (cento e trinta) empregos indiretos (SANTOS; COSTA, 2006).

Para efeito desse estudo optou-se pelo referido consórcio, qual seja: PBTech, por representar um dos modelos de redes empresarias que atua de forma flexível, onde as empresas unem-se para administrar e planejar estratégias de negócios, assim como por sua representativa importância para economia local.

\section{Resultados}

O primeiro momento da apresentação dos resultados está focado em classificar a rede PBTech na tipologia de redes de Hoffmann et. al. (2004), os quais exploram quatro características bases de uma formação em rede: direcionalidade, localidade, formalização e poder de decisão. 
No primeiro indicador - direcionalidade, a rede PBTech pode ser classificada como Horizontal, apesar da heterogeneidade presente entre as empresas participantes, tanto em termos de atividade, tanto em níveis de 'maturidade organizacional'. A rede é constituída, em sua quase totalidade, por pequenas empresas que atuam num mesmo setor e apresentam atividades relacionadas ao desenvolvimento e comercialização de sistemas eletrônicos e computadorizadas ou serviços tecnológicos. As empresas participantes não realizam partes de um mesmo processo produtivo, efetuam sim a produção individual completa, e por isso a rede não é considerada como Vertical. Através de formas associativas e consorciativas, a PBTech visa atingir benefícios ligados a implementação de exportações, objetivo fim do consórcio. Para tanto, o consórcio desenvolve, de acordo com Santos e Costa (2006), ações específicas com foco na melhoria dos processos de gestão, qualidade e internacionalização.

Em relação ao indicador Localidade, a rede pode ser classificada como Dispersa e como Aglomerada. Dispersa, uma vez que estas empresas estão situadas em cidades distintas do estado, no caso Campina Grande e João Pessoa, onde utilizam-se de canais de comunicação mais avançados que permitem superar tais distâncias, e assim manter a interação na rede. Outra razão para serem assim classificadas é o fato de se unirem pela busca da ampliação de mercado, o internacional, onde, ao longo do projeto, já conseguiram exportar seus produtos para diversos países (SANTOS; COSTA, 2006). A rede PBTech também pode ser caracterizada como Aglomerada, devido às relações de cooperação e colaboração entre as empresas, além de contarem com a presença de instituições de apoio empresarial e entidades especializadas.

No indicador Formalização, a rede pode ser classificada como de Base Não-Contratual por apresentar laços de cooperação e sinergias coletivas como fontes de fortalezas e de criação de cadeias de valor usadas em conjunto com estratégias de internacionalização, visualizadas em vários momentos, como na formação de grupos de discussão, para verificação de percepções, discussão de situações específicas já vivenciadas pelos participantes e troca de experiências.

Por último, no indicador Poder de Decisão, a rede não possui um centro de poder que regule as decisões em relação às demais empresas-membro. Assim, essa rede é classificada como nãoorbital, onde cada parte tem a mesma capacidade de tomada de decisão, uma vez que elas trabalham em cooperação.

No que se refere à tipologia estratégica de Miles e Snow (1978) e a partir das percepções acerca dos dados observados, considera-se que a rede PBTech adota estratégia do tipo Prospectora, uma vez que atua num ambiente bastante inovador e dinâmico, que faz com que as empresas deste setor tendam a estarem em constantes mutações e adaptações, buscando diferencial competitivo pelo potencial de criação de produtos e serviços, e assim investindo ferozmente em pesquisa e desenvolvimento (P\&D). Ao longo do projeto do consórcio, foram mais de 15 novas tecnologias 
disponibilizadas no mercado, em especial no âmbito internacional, mostrando o quanto o grupo prioriza a inovação. De forma mais genérica, pode-se verificar algumas inovações adotadas por estas empresas e / ou produtos que fundamentam o consórcio PBTech.

- Sistema de Sonorização de Portos, atendendo às necessidades de disseminação de informações operacionais, institucionais e de segurança;

- LightBase Architect - sistema que oferece um grande avanço no desenvolvimento de aplicações Web, propiciando de forma simples e sem necessidade de alto nível de domínio técnico o desenvolvimento de aplicação;

- GoldenPortal - software para construção de Portais Corporativos, objetivando consolidar informação departamental, concentrando todos os aplicativos em um único lugar com um único acesso;

- Máquinas destinadas à indústria da reciclagem;

- Plugcell (roteador de ligações entre celulares), principal produto de uma das empresas que, agregou mais 6 soluções, contemplando necessidades em comunicação e segurança eletrônica;

- Memorizer - produto projetado para dar o máximo em produtividade e organização de agendas e compromissos;

- MaxChat - sistema de troca de mensagens eletrônicas.

Além da busca pela inovação de produtos/serviços e processos, a estratégia da rede PBTech é direcionada para a expansão de seus mercados de atuação que também configura a tipologia estratégica adotada pela rede, qual seja: a prospectora. O grande objetivo alvo e coletivo da formação de rede é a promoção do desenvolvimento do mercado de software paraibano através da intensificação do processo de divulgação, comercialização local e nacional, além da exportação dos produtos fabricados no estado.

A partir desses resultados, e considerando as características da rede PBTech, esta apresentou-se de forma ideal no que se refere ao modelo de Hoffmann et. al. (2004), uma vez que apresenta linearidade adequadas entre as tipologias encontradas, no que tange ao tipo de produto e/ ou serviço ofertados: horizontal, dispersa e aglomerada, base não-contratual e não orbital. Dada estas características da rede e o contexto em que as empresas de base tecnológica atuam, caracterizado por forte concorrência e necessidade mais intensa de práticas inovadoras baseada em ações coletivas, o tipo de estratégia mais adequada é a prospectora. Neste caso, é possível inferir que existe um alinhamento entre as características da rede com o tipo de estratégia utilizada. Em função disto, o consórcio tem amplo potencial para tornar-se mais competitivo, na medida em que está apta a atuar em novos e diversificados nichos de mercado. 
Nesse sentido, pode-se constatar alguns progressos acerca do alcance dos objetivos propostos pela formação da rede. $\mathrm{O}$ valor das exportações aumentou significativamente, desde o início do projeto (dezembro de 2002) até dezembro de 2005, sendo de aproximadamente 68\%. Também ocorreu o aumento da base exportadora, expandindo o número de empresas: de uma empresa exportadora para oito empresas até o final de dezembro de 2005.

Estes resultados mostram que, a partir de práticas de cooperação, as empresas conseguiram unir forças e assim, expandir seus mercados, alavancar suas vendas e estimular a livre iniciativa, o empreendedorismo e o profissionalismo, visando o desenvolvimento socioeconômico sustentável da rede em questão.

\section{Considerações finais}

Dada a complexidade do ambiente de negócios, onde a cada dia as empresas se encontram a mercê de constantes mutações e dificuldades, se faz necessário a adoção de novas práticas de atuação, intrinsecamente relacionadas à uma postura mais participativa e colaborativa frente ao mercado globalizado e competitivo.

Nesse sentido, as empresas que formam o consórcio PBTech compreenderam que o trabalho de forma associativa geraria maiores condições para superar as dificuldades inerentes ao mercado quanto à competitividade, assim como ganhariam mais força para atuarem em mercados internacionais.

A partir dos resultados encontrados, a PBTech apresentou uma configuração de rede, assim como a tipologia estratégica adequadas com o setor de atuação, o que viabilizou um significativo crescimento da rede, proporcionado pelos laços de cooperação e apoio mútuo em prol de um objetivo coletivo maior.

Porém, algumas reformulações no que se refere às práticas de cooperação adotadas se fazem necessárias, uma vez que se pôde perceber que estas não acontecem com a freqüência ideal, e se apresentam de maneira tímida, devido, principalmente a pouca cultura de cooperação intrínseca a maior parte das empresas brasileiras atuantes no setor de tecnologia, ocasionada, sobretudo, pela descrença nas formas associativas e cooperativas. Tal falta de cooperação e colaboração pode dificultar as trocas de experiências e de conhecimento, necessários para desenvolvimento e alavancagem econômica da rede como um todo.

\section{Abstract}


The transformations in contexts environmental, politics, social, economical and marketing occurring in the world of the businesses they challenge the organizations in the search for practical best than they sustain them front the intransigent competitiveness of the market current. The nets of companies appear exactly with the intention of they make possible such objective, where based in basic beginnings of cooperation, interaction, helps mutual and trust acts in a strategic way on behalf of a collective competitive sustainable This article has as objective analyzes a consortium of companies of technological base, starting from the characteristics of nets interorganizationals proposed by Hoffmann et al. (2004), comparing the current situation of the net with the ideal situation for the section that sharing enterprises of technological base with performance in Technology of the Information and Communication (TICs). Besides, it verifies the alignment among the characteristics of the net with the type of adopted strategy, using typology of strategies, proposed by Miles and Snow (1978). This work is characterized as exploratory and descriptive led under the form of case study, using as techniques the bibliographical research and the collection of secondary and primary data referring to updated data on the net PBTech. Resulted they showed them as companies of small and medium load, to if they unite in net, and if configuring appropriately in structure terms and strategy front to an adult collective purpose they can differ of form competitive in the market.

Key-words: nets enterprises; strategy; competitiveness.

\section{Referências}

ANDREWS, K. R. The concept of corporate strategy. Homewood: Richard D. Irwin, 1965.

BALESTRIN, A.; VARGAS, L. M.; FAYARD, P. Ampliação Interorganizacional do Conhecimento: o caso das redes de cooperação. READ - Revista Eletrônica de Administração, Porto Alegre, v. 11, n. 1, jan-fev. 2005.

BAlestrin, A.; VARGAS, L. M. A Dimensão Estratégica das Redes Horizontais de PMEs: Teorizações e Evidências. RAC - Revista de Administração Contemporânea. Curitiba, v. 8, p. 203-227, 2004. Edição especial.

BESANKO, D.; DRANOVE, D.; SHANLEY, M. Economics of Strategy. New York, John Wiley\& Sons, 2000.

CÂNDIDO, G. A. Fatores Críticos de Sucesso no Processo de Formação, Desenvolvimento e Manutenção de Redes Interempresariais do tipo Agrupamento Industrial entre Pequenas e Médias Empresas: um estudo comparativo de experiências brasileiras. 2001, 356 f. Tese (Doutorado em Engenharia de Produção) - PPGEP - UFSC, Florianópolis.

CASTELlS, M. A sociedade em rede. 2. ed. São Paulo: Paz e Terra, 1999.

CHILD, J. Organizational structure, environment and performance: the role of strategic choice. Sociology, v.6, n.1, 1972 .

cross ${ }^{\text {ref }}$

Strategic choice in the analysis of action, structure, organizations and environment: retrospect and prospect. Organization Studies, v. 18, n. 1, 1997.

FAYARD, P. O jogo da interação: informação e comunicação em estratégia. Caxias do Sul: EDUCS, 2000.

HOFFMANN, V. E.; MOLINA-MORALES, F. X.; MARTINEZ-FERNANDEZ, M. T. Redes de Empresas: Uma proposta de tipologia para sua Classificação. In: ENCONTRO DA ANPAD, 28., 2004, Curitiba. Anais... Rio de Janeiro: AMPAD, 2004.

MARCON, M. MOINET, N. La stratégie-réseau. Paris : Zéro Heure, 2000.

MILES, R. E.; SNOW, C. C. Organizational strategy, structure, and process. New York: Mc Graw-Hill, 1978. 
NOHRIA, N. Is a network perspective a useful way of studying organizations. In: NOHRIA, Nitin \& ECCLES, Robert G.(ed.). Networks and organizations: structure, form, and action. Boston, Massachusetts: Harvard Business School Press, 1992, p. 1-22.

OLAVE, M. E. L.; AMATO NETO, J. Redes de cooperação produtiva: uma estratégia de competitividade e sobrevivência para pequenas e médias empresas. G \& P - Gestão e Produção. v. 8, n. 3, p. 289-303, dez. 2001.

PBTech. Disponível em: <www.pbtech.com.br>. Acesso em: mar. 2007.

SANTOS, J. A.; COSTA, I. PBTech: Ultrapassando fronteiras com soluções criativas. Análise FIA de Comércio Exterior: 2006.

SEBRAE - Serviço Brasileiro de Apoio às Micros e Pequenas Empresas. Arranjo Produtivo de Tecnologia da Informação e Comunicação da Paraíba. João Pessoa: SEBRAE/PB, 2006.

SILVA FILHO, J. F., CÂNDIDO, G. A. A Formação de Redes Interorganizacionais e a sua Influência nas Tipologias de Estratégia e Decisão do Empreendedor. In: ENCONTRO DO CONSELHO LATINO AMERICANO PARA O DESENVOLVIMENTO DO ENSINO DA ADMINISTRAÇÃO, 27, 2002. Porto Alegre. Anais... CLADEA, 2002.

ZAHRA, S. A., PEARCE II, J. A. Research evidence on the Miles-Snow typology. 1979.

\section{Dados dos autores:}

Nome completo: Patrícia Trindade Caldas

Filiação institucional: Universidade Federal da Paraíba - UFPB

Departamento: Programa de Pós-Graduação em Engenharia de Produção - PPGEP

Função: Mestranda

Endereço completo: Rua Rodrigues Alves, 2143, Bodocongó.

CEP: 58101-291, Campina Grande - PB - BRA.

Telefones para contato: (83) 33334461

E-mail: ptcaldas@yahoo.com.br

Nome completo: Ana Cecília Feitosa de Vasconcelos

Filiação Institucional: Universidade Federal de Campina Grande - UFCG

Departamento: Unidade Acadêmica de Administração e Contabilidade - UAAC

Função: Graduanda

Endereço completo para correspondência (bairro, cidade, estado, país e CEP): Rua Frei Martinho,

113, Conceição. CEP: 58102-433. Campina Grande - PB - BRA.

Telefone para contato: (83)8825-4990

E-mail: acvasconcelos@gmail.com.br

Nome Completo: Gesinaldo Ataíde Cândido

Filiação Institucional: Universidade Federal de Campina Grande - UFCG

Departamento: Administração e Contabilidade 
Função: Professor titular em Administração Geral

Endereço Completo: Rua Esperidião Rosas, 235/703, Expedicionários, CEP: 58041-070, João Pessoa - PB - BRA.

Telefone para contato: (83) 3242-1155

E-mail: gacandido@uol.com.br

Recebido para publicação em: 08/11/2007

Aceito para publicação em: 23/11/2007 